\title{
Control of Sleep-Wake Cycles in Drosophila
}

\author{
Abhishek Chatterjee and François Rouyer
}

\begin{abstract}
Inter-oscillator communication modulates and sustains the circadian locomotor rhythms in flies and rodent animal models. In Drosophila, the multioscillator network that controls sleep-wake cycles includes about 150 clock neurons. A subset of lateral neurons (LNs) expressing the Pigment-dispersing factor (PDF) appears to act as a master clock in constant darkness (DD). In light-dark (LD) cycles, flies show a bimodal distribution of their activity, and the PDF-expressing LNs play a major role in the control of the morning bout of activity. In contrast, a subset of PDF-negative LNs can generate evening activity in the absence of other functional oscillators. How these oscillators interact in a fully functional network to shape the sleep-wake cycle remains debated. The PDF neurons strongly influence the PDF-negative ones in DD and, to a lesser extent, in LD. The extent of hierarchy depends on environmental conditions and the way the dominance of PDF neurons is exerted on the different types of PDF-negative neurons is unclear. The recent discovery of light- and temperature-dependent oscillators in the dorsal neurons (DNs) sheds new light on the circuits that control the Drosophila diurnal behavior and its adaptation to environmental changes.
\end{abstract}

\section{Background}

The fruit fly Drosophila melanogaster displays rest-activity rhythms that rely on a circadian clock located in the brain. In light-dark (LD) cycles, adult flies show a bimodal activity with morning and evening peaks at dawn and dusk. Activity rhythms persist in constant darkness (DD), indicating the circadian nature of this behavior. Like peripheral clocks, the brain clock depends on a molecular feedback loop where the CLOCK (CLK) and CYCLE (CYC) transcriptional factors drive the expression of the PERIOD (PER) and TIMELESS (TIM) proteins that repress CLK/CYC activity. The negative feedback loop operates in about 150 neurons,

\footnotetext{
A. Chatterjee • F. Rouyer $(\bowtie)$

Institut de Neurosciences Paris-Saclay, CNRS/Université Paris Sud, Gif-sur-Yvette, France e-mail: Francois.rouyer@inaf.cnrs-gif.fr

P. Sassone-Corsi, Y. Christen (eds.), A Time for Metabolism and Hormones,

Research and Perspectives in Endocrine Interactions,

DOI 10.1007/978-3-319-27069-2_8
} 
the so-called clock neurons, which account for about $0.1 \%$ of the total CNS neural population. The numerical simplicity of these 150 neurons that form a network is remarkable in comparison to central circuits for other hardwired behaviors such as courtship or learning and memory. The Drosophila clock neuronal network is also remarkably simple in comparison to circadian control circuits in vertebrates, where several brain areas, including the hypothalamus, pituitary gland, pineal gland, olfactory bulb, etc., harbor numerous bona fide clock neurons. This smaller number of neurons offers the potential to manipulate oscillators at the single-cell level in vivo, through well-defined genetic handles.

There are two broad populations within the 150 clock neurons of the fly brain; one population is laterally placed and another is located along the dorsal margin of the brain. The lateral neurons (LNs) lie near the interface of the central brain and the optic lobe and are organized into a ventral cluster that include small (s-LNvs) and large (1-LNvs) cells, a dorsal cluster (LNds) and a posterior cluster (LPNs). The dorsal neurons (DNs) are in turn subdivided into three clusters designated as DN1, DN2 and DN3 (Fig. 1). Such anatomical categorization frequently has neurochemical and functional bases; for example, the four most ventral s-LNvs express the Pigment-dispersing factor (PDF) neuropeptide and promote morning activity in LD. Based on strong functional data, mostly behavioral and some neurophysiological in nature, a wiring diagram of these differentiated clusters of brain clock neurons has begun to materialize over the past 10 years. In the following section we will summarize the logic of organization of this circuit.
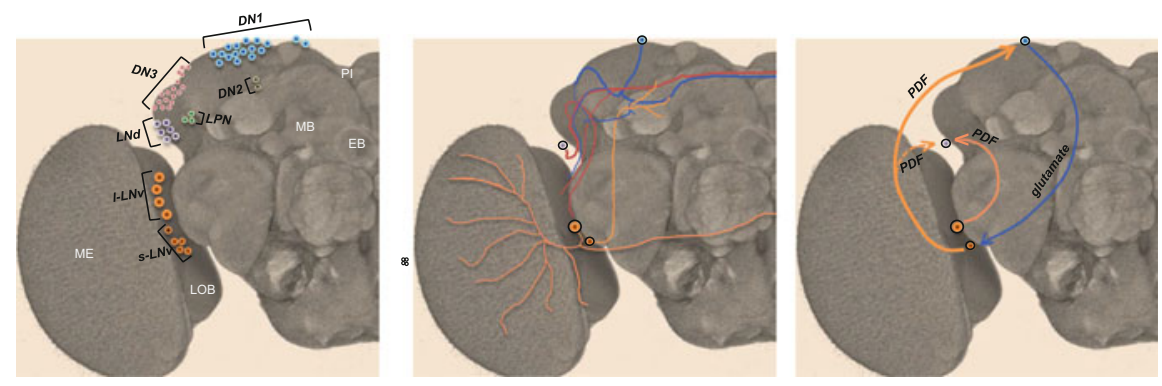

Fig. 1 The clock neurons of the Drosophila brain. Left panel: seven groups of clock neurons have been defined on an anatomical basis. The lateral neurons are organized into a ventral cluster that include small (s-LNvs) and large (1-LNvs) cells, a dorsal cluster (LNds) and a posterior cluster (LPNs). The dorsal neurons include three clusters designated as DN1, DN2 and DN3. Several neuropiles are indicated: Medulla (ME) and Lobula (LOB) in the optic lobe and Pars Intercerebralis (PI), Mushroom Bodies (MB) and Ellipsoid Body (EB) in the central brain. Central panel: projections of the different clock neuron subsets: s-LNvs and 1-LNvs (orange), LNds and fifth PDF-negative s-LNv (red), DN1s (blue). Right panel: communication between neuronal clusters involves PDF from s-LNvs to LNds and DN1s as well as from 1-LNvs to LNds, and glutamate from DN1s to s-LNvs 


\section{Layout of the Network in Constant Conditions}

In the absence of cycling environmental cues, the fly clock circuit has been shown to adopt a functionally minimalist organization. In flies that experienced light-dark cycles and were subsequently kept in constant darkness, the presence of PDF-expressing s-LNvs was required to drive robust rhythmic behavior (Helfrich-Förster 1998; Renn et al. 1999) and a clock restricted to the PDF-expressing cells was sufficient to drive 24-h rhythms (Grima et al. 2004). In contrast, PDF-negative neurons drove behavioral rhythmicity under constant light if light inputs were reduced by the absence of the cell-resident photopigment cryptochrome (CRY). The precise location of these neurons that act as the pacemaker in constant light (LL) has been suggested to be either within the LNd cluster (Picot et al. 2007) or within the DN1s (Murad et al. 2007; Stoleru et al. 2007). In spite of running a functioning oscillator, the s-LNv neurons fail to influence the behavioral period in LL (Picot et al. 2007; Stoleru et al. 2007). The predominant contribution of the s-LNvs to behavioral rhythmicity, as evident in DD, becomes dramatically corroded in LL as ambient light inhibits their behavioral output (Picot et al. 2007). Under constant conditions the operation of the clock circuit remains highly centralized, but depending on the sustained presence or absence of light, this central position is occupied by either the PDF-negative clock neurons or the PDF-positive s-LNv neurons, respectively. Notably, in DD, the s-LNvs operate at the pinnacle of a hierarchy as they enforce a majority of other oscillators to realign their clock program in accordance with the sLNv pace (Stoleru et al. 2005). In contrast, messages from the non-PDF clocks have considerably subdued influence on the running of the master pacemaker in DD (Stoleru et al. 2005; Picot et al. 2007; Collins et al. 2014).

Recently, however, the existence of a centralized monopolar circuit organization in DD has been seriously challenged. The PDF clock has been shown to coherently change behavioral period only over a limited range, which is distributed asymmetrically around the 24-h focal point (Yao and Shafer 2014; Beckwith and Ceriani 2015). When the PDF neurons were forced to run at a pace beyond this specified range, multiple peaks of behavioral period emerged within a single fly, likely as a result of internal desynchronization among multiple oscillators (Yao and Shafer 2014; Beckwith and Ceriani 2015). Because these oscillators are coupled to the PDF clock with differing strength and range of entrainment, they are differentially affected by speed changes in the PDF clock (Yao and Shafer 2014). Thus, the behavioral period in DD is determined by the pace of not only the s-LNv clock but also by other oscillators enjoying different degrees of independence, although they were formerly thought to uniformly behave as slaves of the s-LNv pacemaker. As opposed to direct manipulation of individual oscillator pace, a parallel line of research was to putatively increase the excitability of different subsets of clock neurons to enhance their contribution in the network. This study raised the interesting possibility that the CRY-negative clock neurons, e.g., the DN2s, may have the potential to affect behavioral period like the well-known s-LNvs (Dissel 
et al. 2014). Going one step further, it was proposed that the DD behavioral period is constructed by integrating the slightly longer period dictated by the s-LNvs and the slightly shorter period imposed by the DN2s, with other clock neurons modulating the contributions of these two oscillators (Dissel et al. 2014). The behavioral period would thus depend on the interactions between differently paced oscillators whose endogenous period and influence in the network vary according to environmental conditions. However, speed changes in all clock neurons excluding the PDF cells fell short of altering the behavioral period (Yao and Shafer 2014), in fact bolstering the older idea that PDF oscillators are the predominant determinant of the behavior period in DD. In absence of PDF signaling, the output from the s-LNvs was compromised, thereby allowing secondary oscillators to strongly influence the behavior period (Yao and Shafer 2014). Precisely which oscillators are coupled, whether coupling is directional, how the coupling strength is determined and what are the relative weights of different oscillators to behavioral period according to environmental conditions are some of the questions that fly chronobiologists will probably resolve in next few years. We predict that the existing momentum on neuronal mechanisms of behavioral period determination will be extended to understand the other fundamental parameters of rhythm, such as phase and waveform.

\section{Network Architecture Under LD Cycles}

Depending on the constraints of physiological thermal limit and light availability, animals evolved few basic patterns of diel activity: diurnal, nocturnal, crepuscular or cathemeral (Bennie et al. 2014). In mammals, a given animal can stably and predictably switch back and forth between different patterns in context-dependent ways (Kas and Edgar 1999; Mrosovsky 2003). The choice of a temporal niche takes place downstream of the suprachiasmatic nucleus ( $\mathrm{SCN}$ ) clock and is strongly influenced by light inputs (Mrosovsky and Hattar 2005; Doyle et al. 2008). A comparable plasticity is observed in flies. For example, a typically crepuscular male fly will become nocturnal in the presence of a mate or during moonlit nights (Bachleitner et al. 2007; Fujii et al. 2007) and will be more diurnal when daylight is low (Schlichting et al. 2015). At first glance, the similar phasing of molecular oscillations in the different clock neuron subsets of the brain suggests that shaping the sleep-wake cycle occurs downstream of the clock, but results obtained from manipulating these different subsets support a more complex model.

Flies in the standard laboratory condition of 12:12 LD cycles show a bimodal profile with peaks of activity coinciding with the putative twilight transitions. Very nicely, the behavioral sub-routines of generating a morning peak and an evening peak are orchestrated by two separable subsets of oscillator neurons, the s-LNvs and the LNds, respectively, providing concrete experimental support for the dualoscillator model of Daan and Pittendrigh (Pittendrigh and Daan 1976; Grima et al. 2004; Stoleru et al. 2004) (Fig. 2). Of note, this dual-oscillator ground plan 

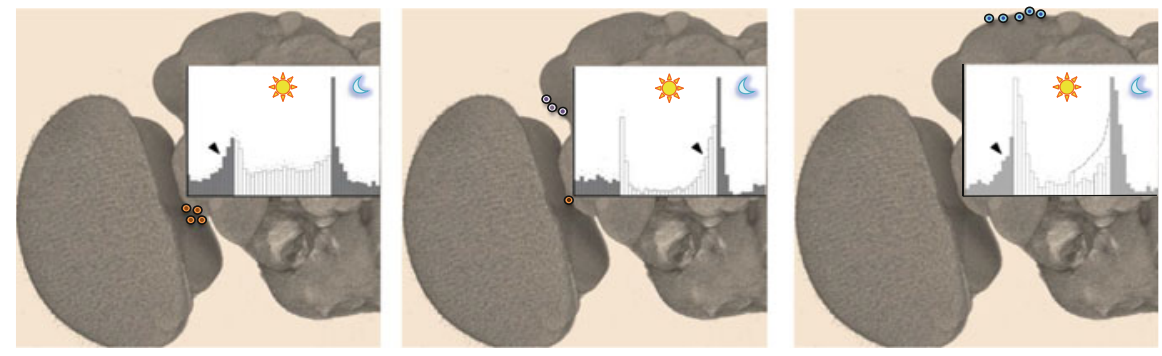

Fig. 2 Contribution of different clock neuron subsets to the LD behavior. Activity plots show the contribution of neuronal groups to morning and evening LD behavior. s-LNvs promote morning activity (left) whereas LNds and the fifth PDF-negative s-LNv promote evening activity (center). DN1s can promote morning activity and evening activity (right). The evening output of the DN1s is very weak in high light but strongly increases in low light (dashed line)

contrasts with the predominantly monopolar hierarchical organization prevailing in DD. The rather autonomous operation of two oscillators under LD cycles is abetted by their independent access to light information through redundant pathways-inhouse CRY signaling and the visual system-whose output impinges on the clock circuit probably at multiple nodes, including the LNvs (Cusumano et al. 2009; Zhang et al. 2009). However, network interaction between oscillator neuron clusters could still be evident in LD. In the absence of PDF secretion by the LNvs, the evening peak of activity is advanced by a couple of hours (Renn et al. 1999). In the absence of both CRY and PDF, the evening peak vanishes and the phase of the molecular oscillations in the LNds is strongly altered (Cusumano et al. 2009; Zhang et al. 2009; Im et al. 2011). The phasing of the evening activity thus increasingly depends on PDF signaling when autonomous CRY-dependent photoreception decreases at the end of the day because of less intense and more reddish light. So far, the role of DNs in shaping the LD activity pattern seems to be largely secondary to the LNs (Grima et al. 2004; Stoleru et al. 2004; Zhang et al. 2010a, b). A functional clock restricted to the DN1s is sufficient to drive both morning and evening activity bouts in low light LD conditions, whereas high light permits morning activity only (Zhang et al. 2010b). These outputs are affected by temperature, and the DN1 neurons thus appear to be capable of integrating certain light and temperature information from the ambient environment (Zhang et al. 2010a, b). Although the expression of PDFR in the DN1s is important for their proper function (Zhang et al. 2010a), how they modulate the clock network's collective output remains unknown.

In summary, under periodic environmental cues, multiple, highly autonomous oscillators with distinct behavioral contributions collaboratively sculpt the organism's activity profile. In line with the 'internal coincidence model' of photoperiodism (Pittendrigh and Minis 1964), flexible changes in the clock network favoring the contribution of particular oscillators under certain ambient environmental conditions have been put forward as the mechanistic basis of seasonal adaptation in flies (Stoleru et al. 2007). The Daan/Pittendrigh model proposed that light 
accelerates morning oscillators and decelerates evening oscillators to adapt the bimodal activity to the changing photoperiod. Fast- and slow-running neuronal oscillators were described in flies displaying split rhythms in LL (Yoshii et al. 2004). However, short and long period components were observed to derive from the LD evening bout, suggesting that light-accelerated clock neurons contribute to the evening activity (Yoshii et al. 2004; Rieger et al. 2006), in contrast to the prediction of the model. As indicated above, light was shown to promote the output of the LNd-based evening oscillator while inhibiting the morning oscillator carried by the PDF-expressing s-LNvs (Picot et al. 2007). The importance of PDF-negative cells in the presence of light is also shown by experiments comparing the relative influence of PDF-positive and PDF-negative neuronal subsets in different photoperiods. This work was done by looking at morning and evening activity peaks of flies with accelerated PDF-positive or PDF-negative neurons. Under long photoperiods, the evening oscillator located in PDF-negative cells was proposed to control the speed of the morning oscillators, whereas in short photoperiod conditions the morning oscillator of PDF cells would take the lead (Stoleru et al. 2007). The discovery of other subsets contributing to morning and evening activity bouts, in particular the DN1s contributing to evening activity in low light only, suggest that the adaptation to photoperiod changes might be more complex. Indeed, we have data indicating that new groups of oscillators are recruited when flies are confronted with summer-like conditions. Such laboratory-based simplified environmental parameters are probably inadequate to explain the working of the network under the complex natural conditions that exist in the spatiotemporal niche inhabited by Drosophila in the wild (Menegazzi et al. 2012, 2013; Vanin et al. 2012; De et al. 2013). In particular, daily temperature variations have a strong impact on the sleep-activity pattern and can even induce some morning and evening anticipatory activity in clockless flies (Vanin et al. 2012; Menegazzi et al. 2013). But the principles and logic of circuit operation learned from a severely artificial set-up could well be valid and applicable for the same network's functionality under more complex natural conditions.

Open Access This chapter is distributed under the terms of the Creative Commons AttributionNoncommercial 2.5 License (http://creativecommons.org/licenses/by-nc/2.5/) which permits any noncommercial use, distribution, and reproduction in any medium, provided the original author(s) and source are credited.

The images or other third party material in this chapter are included in the work's Creative Commons license, unless indicated otherwise in the credit line; if such material is not included in the work's Creative Commons license and the respective action is not permitted by statutory regulation, users will need to obtain permission from the license holder to duplicate, adapt or reproduce the material. 


\section{References}

Bachleitner W, Kempinger L, Wulbeck C, Rieger D, Helfrich-Forster C (2007) Moonlight shifts the endogenous clock of Drosophila melanogaster. Proc Natl Acad Sci USA 104:3538-3543

Beckwith EJ, Ceriani MF (2015) Experimental assessment of the network properties of the Drosophila circadian clock. J Comp Neurol 523:982-996

Bennie JJ, Duffy JP, Inger R, Gaston KJ (2014) Biogeography of time partitioning in mammals. Proc Natl Acad Sci USA 111:13727-13732

Collins B, Kaplan HS, Cavey M, Lelito KR, Bahle AH, Zhu Z, Macara AM, Roman G, Shafer OT, Blau J (2014) Differentially timed extracellular signals synchronize pacemaker neuron clocks. PLoS Biol 12:e1001959

Cusumano P, Klarsfeld A, Chélot E, Picot M, Richier B, Rouyer F (2009) PDF-modulated visual inputs and cryptochrome define diurnal behavior in Drosophila. Nat Neurosci 12:1427-1433

De J, Varma V, Saha S, Sheeba V, Sharma VK (2013) Significance of activity peaks in fruit flies, Drosophila melanogaster, under seminatural conditions. Proc Natl Acad Sci USA 110: 8984-8989

Dissel S, Hansen CN, Ozkaya O, Hemsley M, Kyriacou CP, Rosato E (2014) The logic of circadian organization in Drosophila. Curr Biol 24:2257-2266

Doyle SE, Yoshikawa T, Hillson H, Menaker M (2008) Retinal pathways influence temporal niche. Proc Natl Acad Sci USA 105:13133-13138

Fujii S, Krishnan P, Hardin P, Amrein H (2007) Nocturnal male sex drive in Drosophila. Curr Biol 17:244-251

Grima B, Chélot E, Xia R, Rouyer F (2004) Morning and evening peaks of activity rely on different clock neurons of the Drosophila brain. Nature 431:869-873

Helfrich-Förster C (1998) Robust circadian rhythmicity of Drosophila melanogaster requires the presence of lateral neurons: a brain-behavioral study of disconnected mutants. J Comp Physiol A Neuroethol Sens Neural Behav Physiol 182:435-453

Im SH, Li W, Taghert PH (2011) PDFR and CRY signaling converge in a subset of clock neurons to modulate the amplitude and phase of circadian behavior in Drosophila. PLoS One 6:e18974

Kas MJ, Edgar DM (1999) A nonphotic stimulus inverts the diurnal-nocturnal phase preference in Octodon degus. J Neurosci 19:328-333

Menegazzi P, Yoshii T, Helfrich-Forster C (2012) Laboratory versus nature: the two sides of the Drosophila circadian clock. J Biol Rhythms 27:433-442

Menegazzi P, Vanin S, Yoshii T, Rieger D, Hermann C, Dusik V, Kyriacou CP, Helfrich-Forster C, Costa R (2013) Drosophila clock neurons under natural conditions. J Biol Rhythms 28:3-14

Mrosovsky N (2003) Beyond the suprachiasmatic nucleus. Chronobiol Int 20:1-8

Mrosovsky N, Hattar S (2005) Diurnal mice (Mus musculus) and other examples of temporal niche switching. J Comp Physiol A Neuroethol Sens Neural Behav Physiol 191:1011-1024

Murad A, Emery-Le M, Emery P (2007) A subset of dorsal neurons modulates circadian behavior and light responses in Drosophila. Neuron 53:689-701

Picot M, Cusumano P, Klarsfeld A, Ueda R, Rouyer F (2007) Light activates output from evening neurons and inhibits output from morning neurons in the Drosophila circadian clock. PLoS Biol 5:e315

Pittendrigh C, Daan S (1976) A functional analysis of circadian pacemakers in nocturnal rodents. V Pacemaker structure: a clock for all seasons. J Comp Physiol A Neuroethol Sens Neural Behav Physiol 106:333-335

Pittendrigh CS, Minis DH (1964) The entrainment of circadian oscillations by light and their role as photoperiodic clocks. Am Nat 98:261-294

Renn SC, Park JH, Rosbash M, Hall JC, Taghert PH (1999) A pdf neuropeptide gene mutation and ablation of PDF neurons each cause severe abnormalities of behavioral circadian rhythms in Drosophila. Cell 99:791-802

Rieger D, Shafer OT, Tomioka K, Helfrich-Forster C (2006) Functional analysis of circadian pacemaker neurons in Drosophila melanogaster. J Neurosci 26:2531-2543 
Schlichting M, Grebler R, Menegazzi P, Helfrich-Forster C (2015) Twilight dominates over moonlight in adjusting Drosophila's activity pattern. J Biol Rhythms 30:117-128

Stoleru D, Peng P, Agosto J, Rosbash M (2004) Coupled oscillators control morning and evening locomotor behavior of Drosophila. Nature 431:862-868

Stoleru D, Peng Y, Nawathean P, Rosbash M (2005) A resetting signal between Drosophila pacemakers synchronizes morning and evening activity. Nature 438:238-242

Stoleru D, Nawathean P, Fernandez Mde L, Menet JS, Ceriani MF, Rosbash M (2007) The Drosophila circadian network is a seasonal timer. Cell 129:207-219

Vanin S, Bhutani S, Montelli S, Menegazzi P, Green EW, Pegoraro M, Sandrelli F, Costa R, Kyriacou CP (2012) Unexpected features of Drosophila circadian behavioural rhythms under natural conditions. Nature 484:371-375

Yao Z, Shafer OT (2014) The Drosophila circadian clock is a variably coupled network of multiple peptidergic units. Science 343:1516-1520

Yoshii T, Funada Y, Ibuki-Ishibashi T, Matsumoto A, Tanimura T, Tomioka K (2004) Drosophila cry(b) mutation reveals two circadian clocks that drive locomotor rhythm and have different responsiveness to light. J Insect Physiol 50:479-488

Zhang L, Lear BC, Seluzicki A, Allada R (2009) The CRYPTOCHROME photoreceptor gates PDF neuropeptide signaling to set circadian network hierarchy in Drosophila. Curr Biol 19: 2050-2055

Zhang L, Chung BY, Lear BC, Kilman VL, Liu Y, Mahesh G, Meissner RA, Hardin PE, Allada R (2010a) DN1(p) Circadian neurons coordinate acute light and PDF inputs to produce robust daily behavior in Drosophila. Curr Biol 20:591-599

Zhang Y, Liu Y, Bilodeau-Wentworth D, Hardin PE, Emery P (2010b) Light and temperature control the contribution of specific DN1 neurons to Drosophila circadian behavior. Curr Biol 20:600-605 